\title{
Beam angle optimization for intensity-modulated radiation therapy using a guided pattern search method
}

\author{
Humberto Rocha ${ }^{1}$, Joana M Dias ${ }^{1,2}$, Brígida C Ferreira ${ }^{3,4}$ and \\ Maria C Lopes ${ }^{3,4}$ \\ ${ }^{1}$ INESC-Coimbra, Coimbra, Portugal \\ ${ }^{2}$ Faculdade de Economia, Universidade de Coimbra, Coimbra, Portugal \\ ${ }^{3}$ I3N, Departamento de Física, Universidade de Aveiro, Aveiro, Portugal \\ ${ }^{4}$ Serviço de Física Médica, IPOC-FG, EPE, Coimbra, Portugal \\ E-mail: hrocha@mat.uc.pt
}

\begin{abstract}
Generally, the inverse planning of radiation therapy consists mainly in the fluence optimization. The beam angle optimization (BAO) in intensitymodulated radiation therapy (IMRT) consists of selecting appropriate radiation incidence directions and may influence the quality of the IMRT plans, both to enhance better organs sparing and to improve tumor coverage. However, in clinical practice, most of the time, beam directions continue to be manually selected by the treatment planner without objective and rigorous criteria. The goal of this paper is to introduce a novel approach that uses beam's-eye-view dose ray tracing metrics within a pattern search methods framework in the optimization of the highly non-convex BAO problem. Pattern search methods are derivative-free optimization methods that require few function evaluations to progress and converge and have the ability to better avoid local entrapment. The pattern search methods framework is composed by a search step and a poll step at each iteration. The poll step performs a local search in a mesh neighborhood and assures convergence to a local minimizer or stationary point. The search step provides the flexibility for a global search since it allows searches away from the neighborhood of the current iterate. Beam's-eye-view dose metrics assign a score to each radiation beam direction and can be used within the pattern search framework furnishing a priori knowledge of the problem so that directions with larger dosimetric scores are tested first. A set of clinical cases of head-and-neck tumors treated at the Portuguese Institute of Oncology of Coimbra is used to discuss the potential of this approach in the optimization of the BAO problem.
\end{abstract}




\section{Introduction}

Intensity-modulated radiation therapy (IMRT) is a modern technique where the radiation beam is modulated by a multileaf collimator allowing the irradiation of the patient using non-uniform radiation fields from selected angles aiming to deliver a dose of radiation to the tumor minimizing the damages on the surrounding healthy organs and tissues. The IMRT treatment planning is usually a sequential process where initially a given number of beam directions are selected followed by the fluence map optimization (FMO) at those beam directions. Beam angle optimization (BAO) consists on the selection of appropriate radiation incidence directions and may influence the quality of the IMRT plans, both to enhance organs sparing and to improve tumor coverage. Regardless the evidence presented in the literature that appropriate radiation beam incidence directions can lead to a plan's quality improvement (Aleman et al 2009, Liu et al 2006, Voet et al 2012), in clinical practice, most of the time, beam directions continue to be manually selected by the treatment planner in a time-consuming trial and error iterative process.

Except for rare exceptions, where the BAO problem is addressed as a non-convex nonlinear problem, see, e.g., Craft (2007), for the vast majority of previous work on beam angle optimization, the entire range $\left[0^{\circ}, 360^{\circ}\right]$ of gantry angles is discretized into equally spaced beam directions with a given angle increment, such as 5 or 10 degrees, where exhaustive searches are performed directly or guided by a variety of different heuristics including simulated annealing (Bortfeld and Schlegel 1993, Djajaputra et al 2003, Lu et al 1997, Rowbottom et al 1999), genetic algorithms (Ehrgott et al 2008, Ezzell 1996, Haas et al 1998, Li et al 2004), particle swarm optimization (Li et al 2005) or other heuristics incorporating a priori knowledge of the problem (D'Souza et al 2004, Pugachev and Xing 2002). Although those global heuristics can theoretically avoid local optima, globally optimal or even clinically better solutions can not be obtained without a large number of objective function evaluations. On the other hand, the concept of beam's-eye-view has been a popular approach to address the BAO problem as well (Goitein et al 1983, Lu et al 1997, Pugachev and Xing 2001a). This approach uses topographic criteria to rank the candidate beam directions. For IMRT, the geometrical considerations are not as important. Some variations of the beam's-eye-

view concept consider dosimetric criteria to rank the candidate beam directions selecting those with higher scores (Pugachev and Xing 2001b, Pugachev and Xing 2002). Despite the computational time efficiency of these approaches, the optimality of the solutions proposed cannot be guaranteed since the interplay between the selected beam directions is ignored.

Many of the previous BAO studies are based on a variety of scoring methods or approximations of the FMO to gauge the quality of the beam angle set. However, when the BAO problem is not based on the optimal FMO solutions, the resulting beam angle set has no guarantee of optimality and has questionable reliability since it has been extensively reported that optimal beam angles for IMRT are often non-intuitive (Stein 
et al 1997). Here, similarly to Aleman et al (2008), Craft (2007), Das and Marks (1997), Haas et al (1998), Lim et al (2008), Mišić et al (2010), Schreibmann et al (2004), the optimal solution of the FMO problem was used to drive our BAO problem. The BAO problem is quite difficult since it is a highly non-convex optimization problem with many local minima (Craft 2007, Söderstrom and Brahme 1993). Therefore, methods that avoid being easily trapped in local minima should be used. Obtaining the optimal solution for a beam angle set is time costly and even if only one beam angle is changed in that set, a complete dose computation is required in order to compute and obtain the corresponding optimal FMO solution. To minimize this time issue, methods that require few function value evaluations should be used to tackle the BAO problem.

The incorporation of beam's-eye-view dose metrics information into IMRT and arc therapy treatment planning has been reported with success (Ma et al 2009) and we aim to embed that information in a optimization framework instead of a direct application. The objective of this paper is to introduce a novel approach that uses beam's-eye-view dose ray tracing metrics within a pattern search methods framework in the optimization of the highly non-convex BAO problem. Pattern search methods are derivative-free optimization methods that require few function value evaluations to progress and converge and have the ability to avoid local entrapment. The pattern search methods framework is composed by a search step and a poll step at each iteration. The poll step performs a local search in a mesh neighborhood and assures convergence to a local minimizer or stationary point. The search step provides the flexibility for a global search since it allows searches away from the neighborhood of the current iterate. Beam's-eye-view dose metrics assign a score to each radiation beam direction and can be used in the poll step to rank the neighborhood directions so that directions with larger dosimetric scores are tested first. A set of clinical cases of head-and-neck tumors treated at the Portuguese Institute of Oncology of Coimbra is used to discuss the potential of this approach in the optimization of the BAO problem.

\section{Methods and materials}

In order to model the $\mathrm{BAO}$ problem as a mathematical programming problem, a quantitative measure to compare the quality of different sets of beam angles is required. For the reasons presented before, our approach for modelling the BAO problem uses the optimal solution value of the FMO problem as measure of the quality of a given beam angle set. Many authors consider non-coplanar angles (Aleman et al 2009, Breedveld et al 2012, Das and Marks 1997, Lu et al 1997, Meedt et al 2003, Mišić et al 2010) which result in potentially improved treatment plans (Pooter et al 2006, Wang et al

2005). However, despite the fact that almost every angle is possible for radiation delivery, the use of coplanar angles is predominant. For simplicity, only coplanar angles will be considered. 


\subsection{BAO model}

Let us consider $k$ to be the fixed number of (coplanar) beam directions, i.e., $k$ beam angles are chosen on a circle centered on the CT-slice of the body that contains the isocenter (usually the center of mass of the tumor). Typically, the BAO problem is formulated as a combinatorial optimization problem in which a specified number of beam angles is to be selected among a beam angle candidate pool. The entire range $\left[0^{\circ}, 360^{\circ}\right]$ of gantry angles is generally discretized into equally spaced directions with a given angle increment, such as 5 or 10 degrees. We could think of all possible combinations of $k$ beam angles as an exhaustive global search method. However, this requires an enormous amount of time to calculate and compare all dose distributions for all possible angle combinations. Therefore, an exhaustive search of a large-scale combinatorial problem is considered to be too slow and inappropriate for a clinical setting. Many heuristics and meta-heuristics have been presented as an attempt to reduce the number of combinations to compare. However, most require a prohibitive number of function evaluations when the measure considered is the optimal value of the FMO problem.

A different approach for the formulation of the BAO problem is proposed here. The entire range $\left[0^{\circ}, 360^{\circ}\right]$ of gantry angles will be considered instead of a discretized sample. Since for $\alpha, \beta \in\left[0^{\circ}, 360^{\circ}\right]$, the angle $360^{\circ}+\alpha$ is the same as the angle $\alpha \in\left[0^{\circ}, 360^{\circ}\right]$ and the angle $-\beta$ is equivalent to the angle $360^{\circ}-\beta \in\left[0^{\circ}, 360^{\circ}\right]$, we can avoid a bounded formulation. A basic formulation for the BAO problem is obtained by selecting an objective function such that the best set of beam angles is obtained for the function's minimum:

$$
\begin{array}{ll}
\min & f\left(\theta_{1}, \ldots, \theta_{k}\right) \\
\text { s.t. } & \left(\theta_{1}, \ldots, \theta_{k}\right) \in \mathbb{R}^{k} .
\end{array}
$$

Here, the objective $f\left(\theta_{1}, \ldots, \theta_{k}\right)$ that measures the quality of the set of beam directions $\theta_{1}, \ldots, \theta_{k}$ is the optimal value of the FMO problem for each fixed set of beam directions. Such functions have numerous local optima, which increases the difficulty of obtaining a good global solution. Thus, the choice of the solution method becomes a critical aspect for obtaining a good solution. Our formulation facilitates the use of a class of solution methods that we consider to be suited to successfully address the BAO problem: pattern search methods.

\subsection{FMO model}

For a given beam angle set, an optimal IMRT plan is obtained by solving the FMO problem - the problem of determining the optimal beamlet weights for the fixed beam angles. Many mathematical optimization models and algorithms have been proposed for the FMO problem, including linear models (Romeijn et al 2003), mixed integer linear models (Lee et al 2003), nonlinear models (Cheong et al 2005), and multi-criteria models (Breedveld et al 2007). 
Radiation dose distribution deposited in the patient needs to be assessed accurately in order to solve the FMO problem, i.e., to determine optimal fluence maps. Each structure's volume is discretized in voxels (small volume elements) and the dose is computed for each voxel using the superposition principle, i.e., considering the contribution of each beamlet. Typically, a dose matrix D is constructed from the collection of all beamlet weights, by indexing the rows of $\mathrm{D}$ to each voxel and the columns to each beamlet, i.e., the number of rows of matrix $\mathrm{D}$ equals the number of voxels $\left(N_{v}\right)$ and the number of columns equals the number of beamlets $\left(N_{b}\right)$ from all beam directions considered. Therefore, using matrix format, we can say that the total dose received by the voxel $i$ is given by $\sum_{j=1}^{N_{b}} D_{i j} w_{j}$, with $D_{i j}$ the unitary dose delivered to voxel $i$ by beamlet $j$ and $w_{j}$ the weight of beamlet $j$. Usually, the total number of voxels considered reaches the tens of thousands, thus the row dimension of the dose matrix is of that magnitude. The size of $D$ originates large-scale problems being one of the main reasons for the difficulty of solving the FMO problem.

Here, we will use a convex penalty function voxel-based nonlinear model (Aleman et al 2008, Yang et al 2004). In this model, each voxel is penalized according to the square difference between the amount of dose received by the voxel and the amount of dose desired/allowed for the voxel. This formulation yields a quadratic programming problem with only linear non-negativity constraints on the fluence values (Romeijn et al 2003):

$$
\begin{array}{ll}
\min _{w} & \sum_{i=1}^{N_{v}} \frac{1}{v_{S}}\left[\underline{\lambda}_{i}\left(T_{i}-\sum_{j=1}^{N_{b}} D_{i j} w_{j}\right)_{+}^{2}+\bar{\lambda}_{i}\left(\sum_{j=1}^{N_{b}} D_{i j} w_{j}-T_{i}\right)_{+}^{2}\right] \\
\text { s.t. } & w_{j} \geq 0, j=1, \ldots, N_{b},
\end{array}
$$

where $T_{i}$ is the desired/allowed dose for voxel $i, \underline{\lambda}_{i}$ and $\bar{\lambda}_{i}$ are the penalty weights of underdose and overdose of voxel $i$, and $(\cdot)_{+}=\max \{0, \cdot\}$. Although this formulation allows unique weights for each voxel, similarly to the implementation in Aleman et al (2008), weights are assigned by structure only so that every voxel in a given structure has the weight assigned to that structure divided by the number of voxels of the structure $\left(v_{S}\right)$. This nonlinear formulation implies that a very small amount of underdose or overdose may be accepted in clinical decision making, but larger deviations from the desired/allowed doses are decreasingly tolerated (Aleman et al 2008).

The FMO model is used as a black-box function. Other models used before for BAO include convex penalty function structure-based approaches (Lim et al 2008) and a variety of linear approaches (Craft 2007, Lim et al 2007). It is beyond the scope of this study to discuss if this formulation of the FMO problem is preferable to others. The conclusions drawn regarding BAO coupled with this nonlinear model are valid also if different FMO formulations are considered. 


\subsection{Beam's-eye-view dose metrics}

The use of beams-eye-view dose metrics (BEVD) to rank the irradiation beam directions was introduced by Pugachev and Xing (2001a), (2001b). Unlike conventional beams-eyeview $(\mathrm{BEV})$ tools that consider only geometric criteria, BEVD evaluate each possible beam direction using a score function that accounts for beam modulation. In IMRT, beam directions are non-intuitive and may have to go through sensitive organs to achieve an optimal compromise between target coverage and organs sparing, which makes the geometrical criteria used by BEV limited. An intensity-modulated beam can intercept a large volume of an organ at risk (OAR) or normal tissue and may not be necessarily a bad beam direction. The dose tolerances of the involved structures should be considered also when constructing a metric for measuring the quality of incident beam directions. Therefore, in IMRT, it is more appropriate to measure the quality of a radiation beam direction using a score function based on dosimetric criteria.

A technique based on sensitive structures tolerance dose as a determinant factor for deliverable target dose was used. A radiation beam direction is preferred if it can deliver more dose to the target without exceeding the tolerance dose of the OARs or normal tissue located on the path of the beam (Pugachev and Xing 2001a, Pugachev and Xing 2001b, Pugachev and Xing 2002). To compute the BEVD score of a given gantry angle, a ray tracing is performed for each beamlet involved, and the OARs along the path of the beamlet are located. The maximum achievable intensity of a beamlet depends on the locations and tolerances of the OARs. The score of the beam is obtained under the assumption of a single incident beam. Initially, an intensity that assures the delivery of a dose equal to or higher than the prescription to every target voxel is assigned to all beamlets. Then, iteratively, beamlet intensities are reduced until tolerance dose for every OAR or normal tissue voxel crossed by the all beamlets is not exceeded. The weight obtained for each beamlet represents the maximum usable intensity of the beamlet without violating the tolerance of some sensitive structure. Finally, a forward dose calculation using the maximum usable beamlet intensities is performed and the score of a given beam direction is computed as follows (Pugachev and Xing 2001a, Pugachev and Xing 2001b, Pugachev and Xing 2002):

$$
S_{k}=\frac{1}{N_{T}} \sum_{i \in \text { Target }}\left(\frac{d_{i k}}{D_{P}^{T}}\right)^{2},
$$

where $N_{T}$ is the number of voxels in the target, $D_{P}^{T}$ is the target prescription dose and $d_{i k}$ is the "maximum" dose delivered to the target voxel $i$ by the radiation beam direction $k$, i.e. the dose delivered to the target voxel $i$ by the radiation beam direction $k$ using a forward dose calculation and the maximum usable beamlet intensities.

The above score is based on an intuitive consideration of the deliverable dose capability to the target of a single beam direction. The optimal beam configuration for an IMRT treatment should balance the BEVD score and the beam interplay as a result of the overlap of radiation fields. Therefore, we used BEVD score as a priori knowledge 
to provide a computationally intelligent algorithm for beam angle optimization. This a priori knowledge of the problem was used by a pattern search methods framework.

\subsection{Pattern search methods incorporating BEVD}

Pattern search methods are directional direct search methods that belong to a broader class of derivative-free optimization methods, such that iterate progression is solely based on a finite number of function evaluations in each iteration, without explicit or implicit use of derivatives. We will briefly describe pattern search methods for unconstrained optimization problems such as the beam angle problem formulated in (1). Similar to other derivative-free optimization methods, when minimizing non-convex functions with a large number of local minima, pattern search methods have the ability to avoid being trapped by the closest local minima of the starting iterate, and find a local minimum in a lowest region, i.e., in a region with lower function values.

Pattern search methods generate a sequence of non-increasing iterates $\left\{x^{k}\right\}$ using positive bases (or positive spanning sets) and moving towards a direction that would produce a function decrease. A positive basis for $\mathbb{R}^{n}$ can be defined as a set of nonzero vectors of $\mathbb{R}^{n}$ whose positive combinations span $\mathbb{R}^{n}$ (positive spanning set), but no proper set does. A positive spanning set contains at least one positive basis. It can be shown that a positive basis for $\mathbb{R}^{n}$ contains at least $n+1$ vectors and cannot contain more than $2 n$ (Davis 1954). Positive basis with $n+1$ and $2 n$ elements are referred to as minimal and maximal positive basis, respectively. Commonly used minimal and maximal positive basis are $[I-e]$, with $I$ being the identity matrix of dimension $n$ and $e=\left[\begin{array}{lll}1 & \ldots & 1\end{array}\right]^{\top}$, and $[I-I]$, respectively.

One of the main features of positive bases (or positive spanning sets), that is the motivation for directional direct search methods, is that, unless the current iterate is at a stationary point, there is always a vector $\mathbf{v}^{i}$ in a positive basis (or positive spanning set) that is a descent direction (Davis 1954), i.e., there is an $\alpha>0$ such

that $f\left(x^{k}+\alpha \mathbf{v}^{i}\right)<f\left(x^{k}\right)$. This is the core of directional direct search methods and in particular of pattern search methods. The notions and motivations for the use of positive bases, its properties and examples can be found in (Alberto et al 2004, Davis 1954).

Pattern search methods framework is briefly presented next. Let us denote by $\mathbf{V}$ the $n \times p$ matrix whose columns correspond to the $p(\geq n+1)$ vectors forming a positive spanning set. Given the current iterate $x^{k}$, at each iteration $k$, the next point $x^{k+1}$, aiming to provide a decrease of the objective function, is chosen from a finite number of candidates on a given mesh $M_{k}$ defined as

$$
M_{k}=\left\{x^{k}+\alpha_{k} \mathbf{V z}: \mathbf{z} \in \mathbb{Z}_{+}^{p}\right\}
$$

where $\alpha_{k}$ is the mesh-size (or step-size) parameter and $\mathbb{Z}_{+}$is the set of nonnegative integers. Pattern search methods are organized around two steps at every iteration. The first step consists of a finite search on the mesh, free of rules, with the goal of finding a new iterate that decreases the value of the objective function at the current iterate. This 
step, called the search step, has the flexibility to use any strategy, method or heuristic, or take advantage of a priori knowledge of the problem at hand, as long as it searches only a finite number of points in the mesh. The search step provides the flexibility for a global search since it allows searches away from the neighborhood of the current iterate, and influences the quality of the local minimizer or stationary point found by the method. If the search step fails to produce a decrease in the objective function, a second step, called the poll step, is performed around the current iterate. The poll step follows stricter rules and, using the concepts of positive bases, attempts to perform a local search in a mesh neighborhood around $x^{k}, \mathcal{N}\left(x^{k}\right)=\left\{x^{k}+\alpha_{k} \mathbf{v}\right.$ : for all $\left.\mathbf{v} \in P_{k}\right\} \subset M_{k}$, where $P_{k}$ is a positive basis chosen from the finite positive spanning set $\mathbf{V}$. For a sufficiently small mesh-size parameter $\alpha_{k}$, the poll step is guaranteed to provide a function reduction, unless the current iterate is at a stationary point (Alberto et al 2004). So, if the poll step also fails to produce a function reduction, the mesh-size parameter $\alpha_{k}$ must be decreased. On the other hand, if the search or the poll steps obtain an improved value for the objective function, the mesh-size parameter is increased or held constant.

The most common choice for the mesh-size parameter update is to half the meshsize parameter at unsuccessful iterations and to keep it or double it at successful ones. Nevertheless, the algorithm has different options for step size update (0-3): 0 if the mesh size is increased in every successful iteration; 1 if the mesh update for successful iterations is based on a sufficient decrease condition, allowing mesh contractions; 2 if the mesh update for successful iterations is based on a sufficient decrease condition, but contractions are not allowed; 3 if the mesh size is maintained in successful iterations, except when two consecutive successful iterations are found using the same direction, where the mesh size is increased. Note that, if the initial mesh parameter is a power of $2,\left(\alpha_{0}=2^{l}, l \in \mathbb{N}\right)$, and the initial point is a vector of integers, using this common mesh update, all iterates will be a vector of integers until the mesh-size parameter becomes inferior to 1 . This possibility is rather interesting for the BAO problem.

Recently, the efficiency of pattern search methods improved significantly by reordering the poll directions according to descent indicators built from simplex gradients (Custódio and Vicente 2007). Here, the poll directions were reordered according to the BEVD scores meaning that directions with higher dosimetric value were tested first. The strategy sketched is tailored for addressing the BAO problem taking advantage of prior knowledge of the problem:

\section{Algorithm 2.1 (PSM framework incorporating BEVD)}

0. Initialization Set $k=0$. Compute BEVD scores for each beam angle. Choose $x^{0} \in \mathbb{R}^{n}, \alpha_{0}>0$, and a positive spanning set $\mathbf{V}$.

1. Search step Evaluate $f$ at a finite number of points in $M_{k}$ with the goal of decreasing the objective function value at $x^{k}$. If $x^{k+1} \in M_{k}$ is found satisfying $f\left(x^{k+1}\right)<f\left(x^{k}\right)$, go to step 4 . Both search step and iteration are declared successful. Otherwise, go to step 2 and search step is declared unsuccessful. 
2. Poll step This step is only performed if the search step is unsuccessful. Reorder the poll directions according to the BEVD scores. If $f\left(x^{k}\right) \leq f(x)$ for every $x$ in the mesh neighborhood $\mathcal{N}\left(x^{k}\right)$, then go to step 3 and shrink $M_{k}$. Both poll step and iteration are declared unsuccessful. Otherwise, choose a point $x^{k+1} \in \mathcal{N}\left(x^{k}\right)$ such that $f\left(x^{k+1}\right)<f\left(x^{k}\right)$ and go to step 4 . Both poll step and iteration are declared successful.

3. Mesh reduction Let $\alpha_{k+1}=\frac{1}{2} \times \alpha_{k}$. Set $k=k+1$ and return to step 1 .

4. Mesh expansion Let $\alpha_{k+1}=\alpha_{k}$. Set $k=k+1$ and return to step 1 .

Typically, the stopping criteria of the pattern search methods is based either on the maximum number of function value evaluations allowed or in convergence criteria related with the mesh size. Pattern search methods have the ability to converge globally (Alberto et al 2004), i.e., from arbitrary points, to local minimizers candidates. Despite the nonexistence of any supporting theory, we recall that, due to their blindness caused by the nonuse of derivatives, and also by the flexibility of the search step to incorporate global search procedures while the poll step continues to assure convergence to local minima, numerical evidence about the capability of pattern search methods to compute global minimizers has been reported - see, e.g., Custódio et al (2010) and Alberto et al (2004).

Adding to the efficiency provided by an insightful reordering of the poll directions, the search step is provided with the use of minimum Frobenius norm quadratic models to be minimized within a trust region, which can lead to a significant improvement of direct search for smooth, piecewise smooth, and noisy problems (Custódio et al 2010). The prior knowledge of the problem is also included in this step to take advantage of BEVD scores. A trial point is tested by considering the current best beam angle configuration and replacing the beam direction with smallest BEVD score by a beam direction with larger score that is not in the close neighborhood of the remaining beam directions. The prior knowledge of the problem will be further explored in future work with the inclusion of other strategies that take advantage of BEVD scores in this step. Moreover, the choice of the starting configuration can be tailored as well using BEVD scores. For driving the resolution of the BAO problem, we use the last version of SIDPSM (Custódio and Vicente 2007, Custódio et al 2010) which is a MATLAB, The MathWorks Inc (2002) implementation of the pattern search methods that incorporate the use of BEVD scores.

The benefits of our approach in the optimization of the BAO problem are illustrated using a set of clinical examples of head-and-neck cases that are presented next.

\subsection{Head-and-neck clinical examples}

Ten clinical examples of head-and-neck tumors treated at the Portuguese Institute of Oncology of Coimbra (IPOC) were used retrospectively to test the pattern search methods framework proposed. The selected clinical examples were signalized at IPOC as complex cases where proper target coverage and organ sparing, in particular parotid 
Table 1. Tolerance and prescribed doses for all the structures considered for IMRT optimization.

\begin{tabular}{lccc}
\hline Structure & Mean dose & Max dose & Prescribed dose \\
\hline Spinal cord & - & $45 \mathrm{~Gy}$ & - \\
Brainstem & - & $54 \mathrm{~Gy}$ & - \\
Left parotid & $26 \mathrm{~Gy}$ & - & - \\
Right parotid & $26 \mathrm{~Gy}$ & - & - \\
PTV $_{\mathbf{7 0}}$ & - & - & $70.0 \mathrm{~Gy}$ \\
PTV $_{\mathbf{5 9 . 4}}$ & - & - & $59.4 \mathrm{~Gy}$ \\
\hline
\end{tabular}

sparing, proved to be difficult to obtain with the typical 7-beam equispaced coplanar treatment plans. The patients' CT sets and delineated structures were exported via Dicom RT to a freeware computational environment for radiotherapy research (CERR). Since the head-and-neck region is a complex area where, e.g., the parotid glands are usually in close proximity to or even overlapping with the target volume, careful selection of the radiation incidence directions can be determinant to obtain a satisfying treatment plan. In general, the head-and-neck region is a complex area to treat with radiotherapy due to the large number of sensitive organs in this region (e.g., eyes, mandible, larynx, oral cavity, etc.). For simplicity, in this retrospective study, the OARs used for treatment optimization were limited to the spinal cord, the brainstem and the parotid glands.

The spinal cord and the brainstem are some of the most critical OARs in the headand-neck tumor cases. These are serial type organs, i.e., organs such that if only one functional subunit is damaged, the whole organ functionality is compromised. Therefore, if the tolerance dose is exceeded, it may result in functional damage to the whole organ. Thus, it is extremely important not to exceed the tolerance dose assigned for these type of organs. Other than the spinal cord and the brainstem, the parotid glands are also important OARs. The parotid gland is the largest of the three salivary glands. A common complication due to parotid glands irradiation is xerostomia. This secondary radiation effect decreases the quality of life of patients undergoing radiation therapy of head-and-neck, causing difficulties to swallow. The parotids are parallel organs, i.e., if a small volume of the organ is damaged, the organ functionality may not be affected. Their tolerance dose depends strongly on the fraction of the volume irradiated. Hence, if only a small fraction of the organ is irradiated the tolerance dose is much higher than if a larger fraction is irradiated. Thus, for these parallel type structures, the organ mean dose is generally used as an objective for inverse planning optimization.

For the head-and-neck cases in study the planning target volume (PTV) consisted of $P T V_{70}$ and $P T V_{59.4}$ corresponding to different prescribed doses. The prescription dose for the target volumes and tolerance doses for the OARs considered in the optimization are presented in Table 1 . 


\subsection{Computational tests}

Our tests were performed on a 2.66Ghz Intel Core Duo PC with 3 GB RAM. In order to facilitate convenient access, visualization and analysis of patient treatment planning data, as well as dosimetric data input for treatment plan optimization research, the computational tools developed within MATLAB and CERR - computational environment for radiotherapy research (Deasy et al 2003) are used widely for IMRT treatment planning research. We used CERR 3.2.2 version and MATLAB 7.4.0 (R2007a). The dose matrix $D_{i j}$, the unitary dose delivered to voxel $i$ by beamlet $j$, was computed using CERR's pencil beam algorithm. An automatized procedure for dose computation for each given beam angle set was developed, instead of the traditional dose computation available from IMRTP module accessible from CERR's menubar. This automatization of the dose computation was essential for integration in our BAO algorithm. To address the convex nonlinear formulation of the FMO problem we used a trust-region-reflective algorithm (fmincon) of MATLAB 7.4.0 (R2007a) Optimization Toolbox. The FMO problem is inherently a multicriteria optimization problem with conflicting objectives. Despite this convex nonlinear formulation being commonly used for $\mathrm{FMO}$ and $\mathrm{BAO}$, it requires the subjective decision of assigning penalty weights to be used, which is an handicap. In our tests, for all cases, higher penalty weight values were assigned to the target volumes $\left(\underline{\lambda}_{i}=\bar{\lambda}_{i}=4\right)$ compared to the weights assigned to the OARs $\left(\bar{\lambda}_{i}=2\right)$ and the weight assigned to the remaining tissue $\left(\bar{\lambda}_{i}=1\right)$. The penalty weights of underdose were assigned to zero for volumes other than target volumes. The desired/allowed dose for voxel $i, T_{i}$, corresponds to the tolerance or prescribed dose presented in Table 1 except for structures with maximum dose limits where overdose starts to be penalized 5 Gy before the maximum allowed.

We choose to implement the incorporation of BEVD into the pattern search methods framework taking advantage of the availability of an existing pattern search methods framework implementation used successfully by us to tackle the BAO problem (Rocha et al 2012) - the last version of SID-PSM (Custódio et al 2010, Custódio and Vicente 2007). The spanning set used was the positive spanning set ([e-e $I-I]$. Each of these directions corresponds to, respectively, the rotation of all incidence directions clockwise, the rotation of all incidence directions counter-clockwise, the rotation of each individual incidence direction clockwise, and the rotation of each individual incidence direction counter-clockwise. Since we want to improve the quality of the typical equispaced treatment plans, equispaced coplanar beam angles were considered as the initial point for the beam angle optimization process. The choice of this initial point and the non-increasing property of the sequence of iterates generated by SID-PSM imply that each successful iteration correspond to an effective improvement with respect to the usual equispaced beam configuration. The stopping criteria considered is based in convergence criteria related with the mesh size. Since we are interested in integer beam angle directions, the method stops when $\alpha_{k}<1$. The algorithm has different mesh size

update strategies described before. The strategy adopted for this work, that proved to 
be best suited for the problem at hand, consider the maintenance of the mesh size in successful iterations and halve it at unsuccessful ones.

\section{Results}

The present work aims to test the potential of our approach that incorporates a priori knowledge in pattern search methods applied to the optimization of the BAO problem, both in terms of optimal function value and treatment plan improvement. The incorporation of BEVD into the pattern search methods framework was tested using ten clinical examples of head-and-neck tumors treated at IPOC. IMRT treatment plans with seven equispaced coplanar beams were used at IPOC and are commonly used in practice to treat head-and-neck cases (Aleman et al 2008). Therefore, treatment plans of seven coplanar orientations were obtained using our BAO algorithm, denoted $B E V D-P S M$. These plans were compared with the typical 7-beam equispaced coplanar treatment plans denoted equi and with treatment plans obtained with BEVD directly as well, denoted BEVD.

Beforehand, we need to compute the BEVD scores that will be the prior knowledge of the problem to be incorporated in the BAO optimization algorithm. For each patient, the scores for every beam angle were computed as described in Section 2.4, The obtained scores for the first patient are displayed in Fig. 11. Since the solution obtained with BEVD directly considering the peaks of Fig. 1 (Pugachev and Xing 2001a, Pugachev and Xing 2001b) struggled to be competitive with the equi solutions, other configurations were tested as follows. The entire range $\left[0^{\circ}, 360^{\circ}\right]$ of gantry angles is randomly divided in 7 intervals and the configurations tested correspond to the beam angles with maximum scores within each of those intervals, provided that the chosen beam angles are not too close, i.e. correspond to upper and lower limits of two consecutive intervals. The number of configurations tested corresponds to the number of function evaluations required by $B E V D-P S M$ to converge for each case.

The results of $\mathrm{BAO}$ optimization concerning the improvement of the objective function value for the ten clinical cases of head-and-neck tumors using the described BEVD strategy and BEVD-PSM are presented in Table 2, The comparison of the best beam angle configurations obtained by both approaches with the equispaced beam angle configuration in terms of objective function value is clearly favorable to BEVD-PSM. It is important to emphasize the small number of function evaluations required by $B E V D$ $P S M$ to converge. The pattern search approach SID-PSM without the incorporation of a priori knowledge through BEVD scores was also tested using the same parameters for the ten cases. The results in terms of objective function value are slightly worst and both the number of function value evaluations and the computational time are significantly larger.

Despite the improvement in FMO value, the quality of the results can be perceived considering a variety of metrics. A metric clinically used for plan evaluation is the volume of PTV that receives $95 \%$ of the prescribed dose. Typically, $95 \%$ of the PTV 

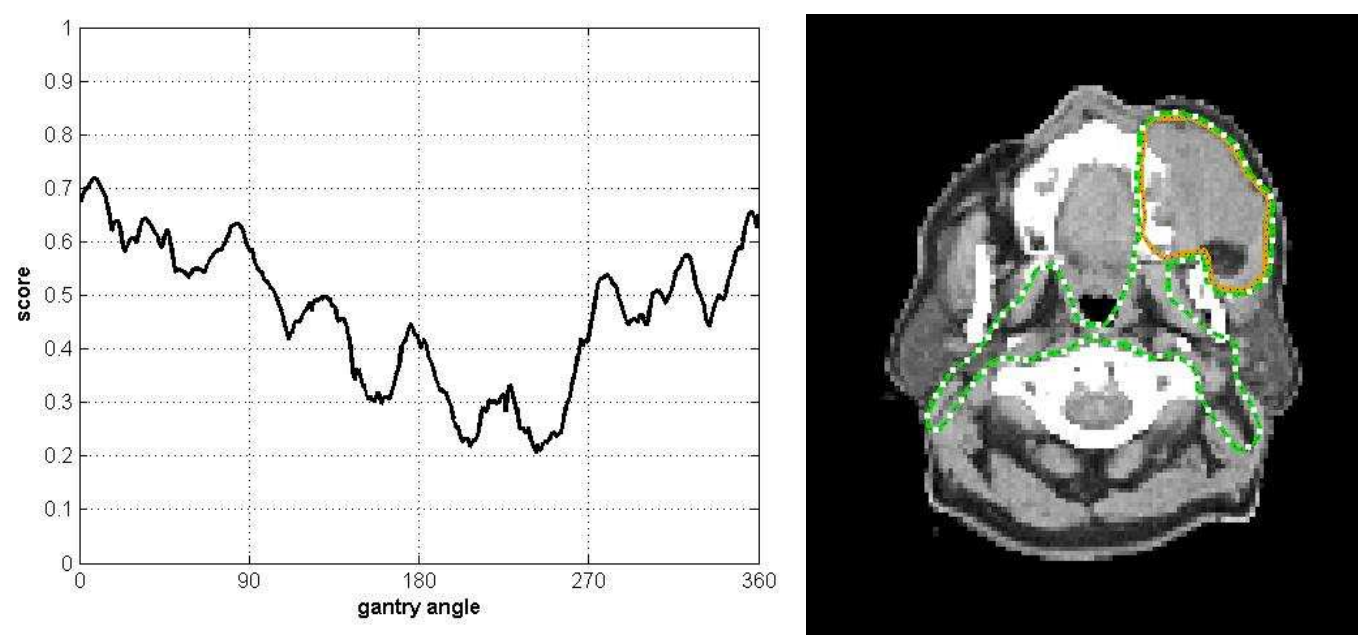

Figure 1. BEVD scores as a function of the gantry angle for the first patient are displayed on the left. On the right, $P T V_{70}$ and $P T V_{59.4}$ of first patient are delineated by a solid and a dotted line, respectively.

Table 2. FMO value improvement obtained by BEVD and BEVD-PSM.

\begin{tabular}{cccccccccc}
\hline & \multirow{2}{*}{ equi } & \multicolumn{3}{c}{$B E V D$} & & \multicolumn{4}{c}{$B E V D-P S M$} \\
\cline { 9 - 10 } \cline { 8 - 9 } Case & Fval & & Fval & \% decrease & & Fval & \% decrease & Fevals & time (h) \\
\hline 1 & 180.8 & 173.9 & $3.8 \%$ & & 162.4 & $10.2 \%$ & 158 & 2.03 \\
2 & 74.8 & 69.8 & $6.7 \%$ & & 66.4 & $11.2 \%$ & 145 & 1.23 \\
3 & 180.5 & 175.1 & $3.0 \%$ & & 157.3 & $12.9 \%$ & 170 & 2.19 \\
4 & 159.4 & 154.8 & $2.9 \%$ & & 144.5 & $9.3 \%$ & 167 & 1.57 \\
5 & 307.9 & 295.1 & $4.2 \%$ & & 280.1 & $9.0 \%$ & 154 & 1.98 \\
6 & 253.2 & 247.7 & $2.2 \%$ & & 232.6 & $8.1 \%$ & 172 & 1.93 \\
7 & 28.7 & 27.2 & $5.2 \%$ & & 24.9 & $13.2 \%$ & 144 & 2.07 \\
8 & 140.4 & 138.1 & $1.6 \%$ & & 126.1 & $10.2 \%$ & 142 & 1.90 \\
9 & 108.1 & 103.1 & $4.6 \%$ & & 97.7 & $9.6 \%$ & 161 & 1.83 \\
10 & 163.6 & 158.4 & $3.2 \%$ & & 148.1 & $9.5 \%$ & 143 & 1.60 \\
\hline
\end{tabular}

volume is required to receive $95 \%$ of the prescribed dose. These metrics are displayed for the ten cases in Fig. 2, The horizontal lines represent $95 \%$ of the prescribed dose. Satisfactory treatment plans should obtain results above these lines. By simple inspection we can verify the advantage of BEVD-PSM treatment plans compared to $B E V D$ and equi treatment plans.

Mean and/or maximum doses for the OARs are displayed for the ten cases in Fig. 3. The horizontal lines represent the tolerance (mean or maximum) dose for the corresponding structures. Thus, satisfactory treatment plans should obtain results under 

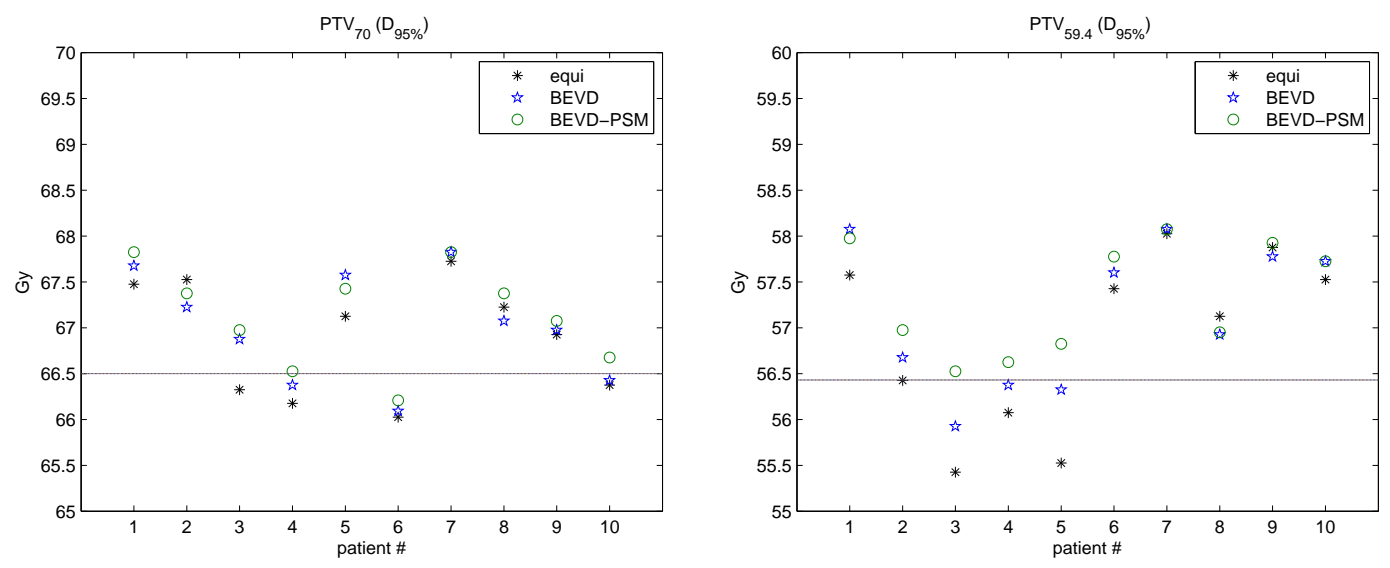

Figure 2. Target irradiation metrics obtained by equi, BEVD and BEVD-PSM.

these lines. For spinal cord and brainstem treatment plans fulfill the maximum dose tolerance in all tested cases. However, as expected, the mean dose limit for the parotids was only achieved few times, mostly by BEVD-PSM treatment plans. Moreover, BEVD$P S M$ treatment plans outperforms $B E V D$ and equi treatment plans in terms of mean dose obtained.

Typically, results are judged also by their cumulative dose-volume histogram (DVH). For illustration, DVH results for the first patient are displayed in Fig. 4. For clarity, the DVHs were split in $P T V_{70}$ and $P T V_{59.4}$ and the remaining structures distributed as an attempt to better visualize the results. The asterisks indicate $95 \%$ of PTV volumes versus $95 \%$ of the prescribed doses. The results displayed in Fig. 4 confirm the benefits of using the optimized beam directions obtained and used in BEVD-PSM treatment plans.

\section{Discussion and conclusions}

The BAO problem is a continuous global highly non-convex optimization problem known to be extremely challenging and yet to be solved satisfactorily. Modern technologies such as arc therapies avoid this problem and are becoming increasingly popular due to the short treatment times and improved dose distributions. However this technology is not yet available to all radiation therapy departments. Gantry angle optimization could then bring improvements to a number of clinics around the world that are limited to the use of fixed gantry directions in IMRT delivery. At this point it is not possible to know how much there is to gain with gantry angular optimization relatively to arc IMRT since investigation is still being carried on towards the development of an algorithm capable of calculating the BAO global optimal solution.

A new approach for the resolution of the BAO problem, incorporating prior knowledge in a pattern search methods framework, was proposed and tested using a set of clinical head-and-neck cases. We have shown that a beam angle set can be improved 

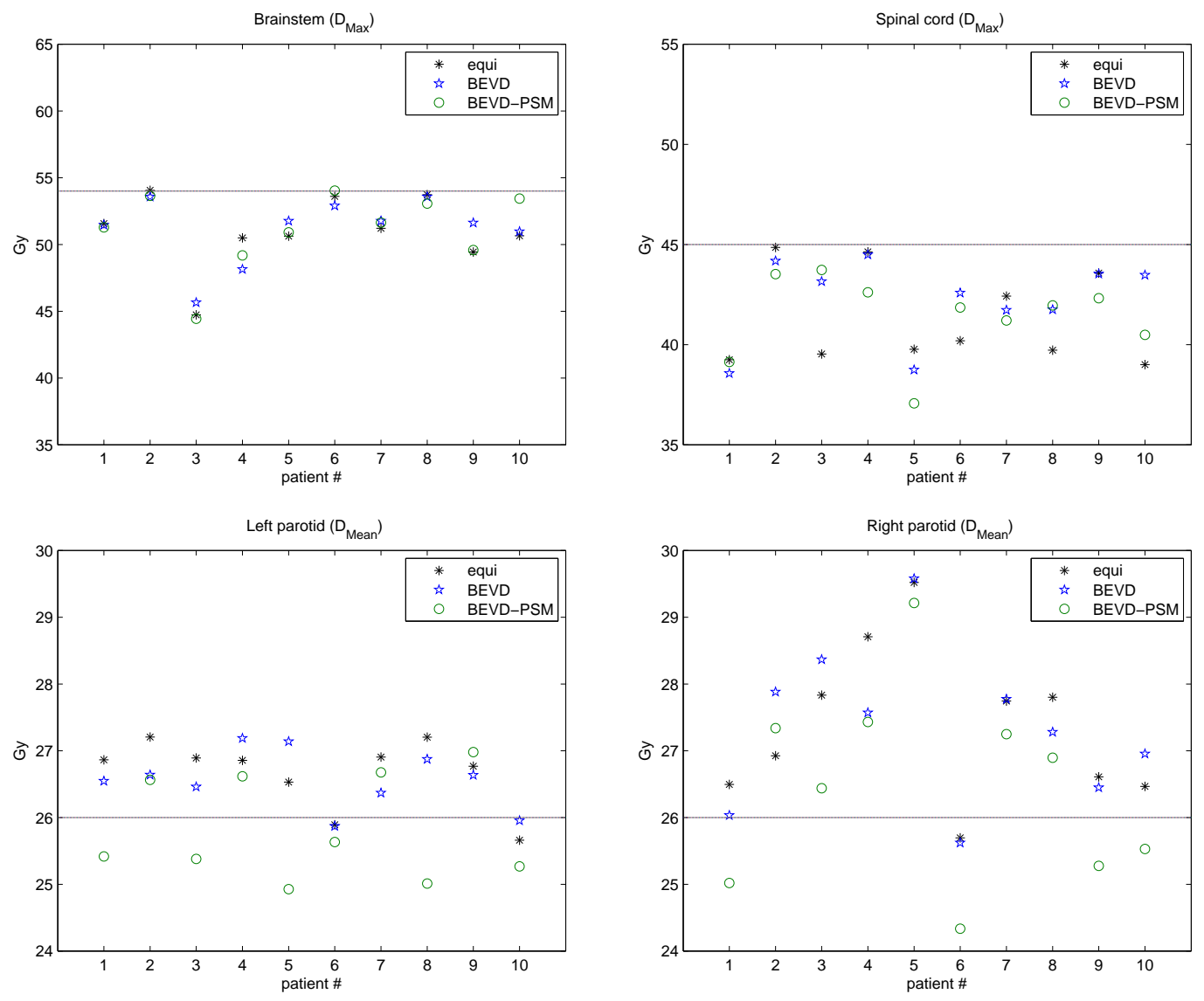

Figure 3. Organ sparing metrics obtained by equi, BEVD and BEVD-PSM.
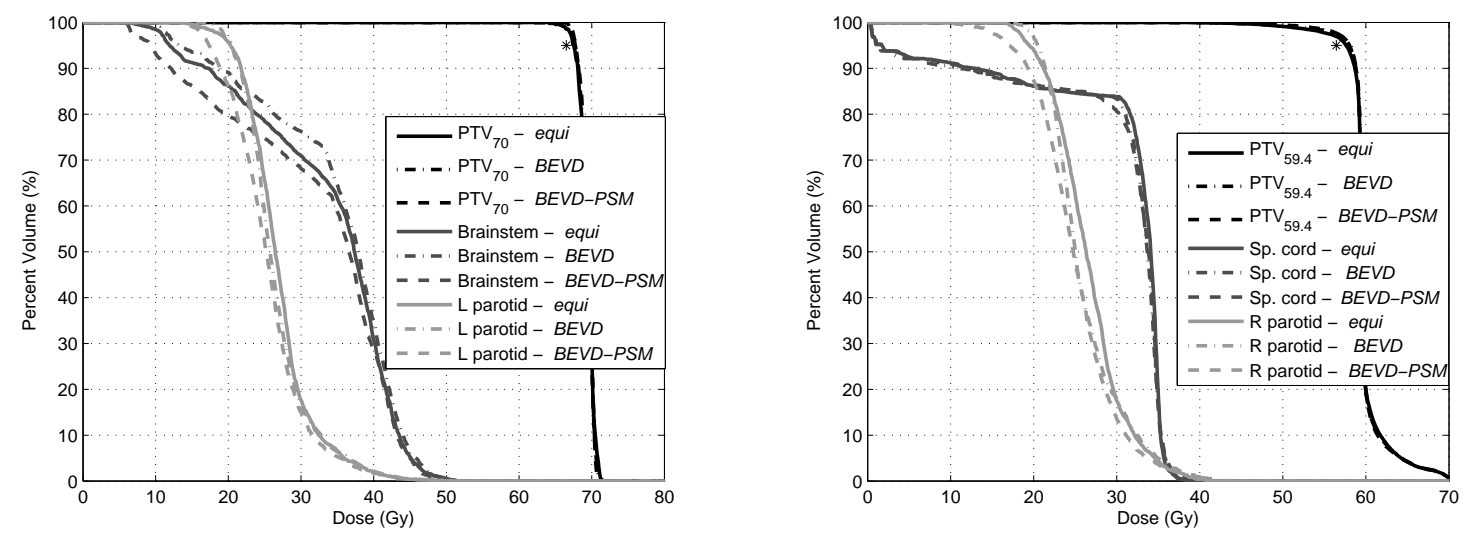

Figure 4. Cumulative dose volume histogram comparing the results obtained by equi, $B E V D$ and BEVD-PSM for the first case.

using our approach. Pattern search methods framework is a suitable approach for the resolution of the non-convex BAO problem due to their structure, organized around two phases at every iteration. The poll step, where convergence to a local minima is assured, and the search step, where flexibility is conferred to the method since any strategy can 
be applied. The poll step is provided with a priori information of the problem which can accelerate the convergence and lead to better solutions since irradiation directions with higher dosimetric potential are tested first. The use of minimum Frobenius norm quadratic models to be minimized within a trust region are used in the search step and can also lead to a significant improvement of direct search for the type of problems at hand. Furthermore, the search step was improved with the incorporation of a tailored strategy making use of BEVD scores. Adding to the search step flexibility, and similarly to other derivative-free optimization methods, when minimizing non-convex functions with a large number of local minima, pattern search methods have the ability to avoid being trapped by the closest local minima of the starting iterate, and find a local minima in lowest regions.

For the clinical cases retrospectively tested, the use of prior knowledge of the patient in our tailored approach showed a positive influence on the quality of the local minimizer found. The improvement of the local solutions in terms of objective function value corresponded, for the head-and-neck cases tested, to high quality treatment plans with better target coverage and with improved organ sparing, in particular better parotid sparing. Moreover, we have to highlight the low number of function evaluations required by BEVD-PSM to converge to a solution. This feature is an advantage that should be even more relevant when considering non-coplanar directions since the number of possible directions to consider increases significantly. The efficiency on the number of function value computations is of the utmost importance, particularly when the BAO problem is modeled using the optimal values of the FMO problem. Moreover, the dose metrics can be further improved namely towards radiobiological criteria.

\section{Acknowledgements}

This work was supported by QREN under Mais Centro (CENTRO-07-0224-FEDER002003) and FEDER funds through the COMPETE program and Portuguese funds through FCT under project grant PTDC/EIA-CCO/121450/2010. This work has also been partially supported by FCT under project grant PEst-C/EEI/UI0308/2011. The work of $\mathrm{H}$. Rocha was supported by the European social fund and Portuguese funds from MCTES.

\section{References}

Alberto P, Nogueira F, Rocha H and Vicente L N 2004 Pattern search methods for user-provided points: Application to molecular geometry problems SIAM J. Optim. 14 1216-36

Aleman D M, Kumar A, Ahuja R K, Romeijn H E and Dempsey J F 2008 Neighborhood search approaches to beam orientation optimization in intensity modulated radiation therapy treatment planning J. Global Optim. 42 587-607

Aleman D M, Romeijn H E and Dempsey J F 2009 A response surface approach to beam orientation optimization in intensity modulated radiation therapy treatment planning INFORMS J. Comput.: Computat. Biol. Med. Appl. 21 62-76 
Bortfeld T and Schlegel W 1993 Optimization of beam orientations in radiation therapy: some theoretical considerations Phys. Med. Biol. 38 291-304

Breedveld S, Storchi P R M, Keijzer M, Heemink A W and Heijmen B J M 2007 A novel approach to multi-criteria inverse planning for IMRT Phys. Med. Biol. 52 6339-53

Breedveld S, Storchi P, Voet P and Heijmen B 2012 iCycle: integrated, multicriterial beam angle, and profile optimization for generation of coplanar and noncoplanar IMRT plans Med. Phys. 39 951-63

Cheong K, Suh T, Romeijn H, Li J and Dempsey J 2005 Fast Nonlinear Optimization with Simple Bounds for IMRT Planning Med. Phys. 321975

Craft D 2007 Local beam angle optimization with linear programming and gradient search Phys. Med. Biol. 52 127-35

Custódio A L and Vicente L N 2007 Using sampling and simplex derivatives in pattern search methods SIAM J. Optim. 18 537-55

Custódio A L, Rocha H and Vicente L N 2010 Incorporating minimum Frobenius norm models in direct search Comput. Optim. Appl. 46 265-78

Das S K and Marks L B 1997 Selection of coplanar or non coplanar beams using three-dimensional optimization based on maximum beam separation and minimized nontarget irradiation Int. J. Radiat. Oncol. Biol. Phys. 38 643-55

Davis C 1954 Theory of positive linear dependence Am. J. Math. 76 733-46

Deasy J O, Blanco A I and Clark V H 2003 CERR: A Computational Environment for Radiotherapy Research Med. Phys. 30 979-85

Djajaputra D, Wu Q, Wu Y and Mohan R 2003 Algorithm and performance of a clinical IMRT beamangle optimization system Phys. Med. Biol. 48 3191-212

D'Souza W D, Meyer R R and Shi L 2004 Selection of beam orientations in intensity-modulated radiation therapy using single beam indices and integer programming Phys. Med. Biol. 49 346581

Ehrgott M, Holder A and Reese J 2008 Beam selection in radiotherapy design Linear Algebra Appl. $4281272-312$

Ezzell G A 1996 Genetic and geometric optimization of three-dimensional radiation therapy treatment planning Med. Phys. 23 293-305

Goitein M, Abrams M, Rowell D, Pollari H and Wiles J 1983 Multidimensional treatment planning: II. Beams eye-view, back projection, and projection through CT sections Int. J. Radiat. Oncol. Biol. Phys. 9 789-97

Haas O C, Burnham K J and Mills J 1998 Optimization of beam orientation in radiotherapy using planar geometry Phys. Med. Biol. 43 2179-93

Yang Y and Xing L 2004 Inverse treatment planning with adaptively evolving voxel-dependent penalty scheme Med. Phys. 31 2839-44

Lee E K, Fox T and Crocker I 2003 Integer programming applied to intensity-modulated radiation therapy treatment planning Ann. Oper. Res. 119 165-81

Li Y, Yao J and Yao D 2004 Automatic beam angle selection in IMRT planning using genetic algorithm Phys. Med. Biol. 49 1915-32

Li Y, Yao D, Yao J and Chen W 2005 A particle swarm optimization algorithm for beam angle selection in intensity modulated radiotherapy planning Phys. Med. Biol. 50 3491-514

Lim G J, Ferris M C, Wright S J, Shepard D M and Earl M A 2007 An optimization framework for conformal radiation treatment planning INFORMS J. Comput. 19 366-80

Lim G J, Choi J and Mohan R 2008 Iterative solution methods for beam angle and fluence map optimization in intensity modulated radiation therapy planning OR Spectrum 30 289-309

Liu H H, Jauregui M, Zhang X, Wang X, Dongand L and Mohan R 2006 Beam angle optimization and reduction for intensity-modulated radiation therapy of non-small-cell lung cancers Int. J. Radiat. Oncol. Biol. Phys. 65 561-72

Lu H M, Kooy H M, Leber Z H and Ledoux R J 1997 Optimized beam planning for linear acceleratorbased stereotactic radiosurgery Int. J. Radiat. Oncol. Biol. Phys. 39 1183-89 
Ma Y, Popple R, Suh T S and Xing L 2009 Beam's-eye-view Dosimetrics-guided inverse planning for aperture-modulated arc therapy Int. J. Radiat. Oncol. Biol. Phys. 75 1587-95

MATLAB, The MathWorks Inc. http://www.mathworks.com

Meedt G, Alber M and Nüsslin F 2003 Non-coplanar beam direction optimization for intensitymodulated radiotherapy Phys. Med. Biol. 48 2999-3019

Mišić V V, Aleman D M and Sharpe M B 2010 Neighborhood search approaches to non-coplanar beam orientation optimization for total marrow irradiation using IMRT Eur. J. Oper. Res. 205 522-27

Pooter J A, Romero A M, Jansen W P A, Storchi P R M, Woudstra E, Levendag P C and Heijmen B J M 2006 Computer optimization of noncoplanar beam setups improves stereotactic treatment of liver tumors Int. J. Radiat. Oncol. Biol. Phys. 66 913-22

Pugachev A and Xing L 2001 Computer-assisted selection of coplanar beam orientations in intensitymodulated radiation therapy Phys. Med. Biol. 46 2467-76

Pugachev A and Xing L 2001b Pseudo beam's-eye-view as applied to beam orientation selection in intensity-modulated radiation therapy Int. J. Radiat. Oncol. Biol. Phys. 51 1361-70

Pugachev A and Xing L 2002 Incorporating prior knowledge into beam orientation optimization in IMRT Int. J. Radiat. Oncol. Biol. Phys. 54 1565-74

Rocha H, Dias J M, Ferreira B C and Lopes M C 2012 Selection of intensity modulated radiation therapy treatment beam directions using radial basis functions within a pattern search methods framework J. Glob. Optim. (at press) doi:10.1007/s10898-012-0002-5

Romeijn H E, Ahuja R K, Dempsey J F, Kumar A and Li J 2003 A novel linear programming approach to fluence map optimization for intensity modulated radiation therapy treatment planing Phys. Med. Biol. 48 3521-42

Rowbottom C G, Oldham M and Webb S 1999 Constrained customization of non-coplanar beam orientations in radiotherapy of brain tumours Phys. Med. Biol. 44 383-99

Schreibmann E, Lahanas M, Xing L and Baltas D 2004 Multiobjective evolutionary optimization of the number of beams, their orientations and weights for intensity-modulated radiation therapy Phys. Med. Biol. 49 747-70

Söderstrom S and Brahme A 1993 Optimization of the dose delivery in a few field techniques using radiobiological objective functions Med. Phys. 20 1201-10

Stein J, Mohan R, Wang X H, Bortfeld T, Wu Q, Preiser K, Ling C C and Schlegel W 1997 Number and orientation of beams in intensity-modulated radiation treatments Med. Phys. 24 149-60

Voet P, Breedveld S, Dirkx M, Levendag P and Heijmen B 2012 Integrated multi-criterial optimization of beam angles and intensity profiles for coplanar and non-coplanar head and neck IMRT and implications for VMAT Med. Phys. 394858

Wang X, Zhang X, Dong L, Liu H, Gillin M, Ahmad A, Ang K, Mohan R 2005 Effectiveness of noncoplanar IMRT planning using a parallelized multiresolution beam angle optimization method for paranasal sinus carcinoma Int. J. Radiat. Oncol. Biol. Phys. 63 594-601 